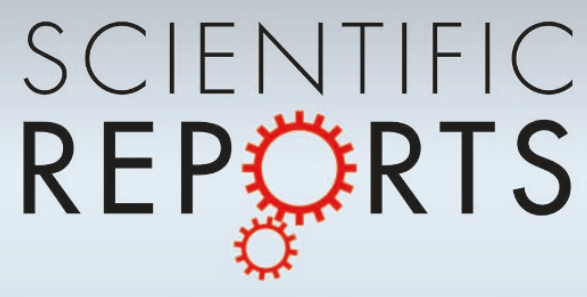

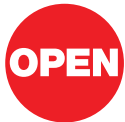

SUBJECT AREAS:

BIOMIMETICS

DISEASES OF THE NERVOUS SYSTEM

CELL DEATH IN THE NERVOUS SYSTEM

ALZHEIMER'S DISEASE

Received

5 December 2012

Accepted

7 May 2013

Published

30 May 2013

Correspondence and requests for materials should be addressed to J.Y.P. (jrpark@cau.ac.

kr) or S.-H.L. (dbiomed@korea.ac.

\title{
Neurotoxic amyloid beta oligomeric assemblies recreated in microfluidic platform with interstitial level of slow flow
}

\author{
Yoon Jung Choi', Sukyung Chae', Jeong Hun Kim' ', Kate F. Barald ${ }^{2}$, Joong Yull Park³ \& Sang-Hoon Lee'
}

\begin{abstract}
'Department of Biomedical Engineering, College of Health Science, Korea University, Seoul, Republic of Korea, ${ }^{2}$ Department of Cell and Developmental Biology, Life Science Institute, University of Michigan, Ann Arbor, MI, USA, ${ }^{3}$ School of Mechanical Engineering, College of Engineering, Chung-Ang University, Seoul, Republic of Korea.
\end{abstract}

\begin{abstract}
Alzheimer's disease is accompanied by progressive, time-dependent changes of three moieties of amyloid beta. In vitro models therefore should provide same conditions for more physiologic studies. Here we observed changes in the number of fibrils over time and studied the correlation between amyloid beta moieties and neurotoxicity. Although the number of fibrils increased dramatically, the change in neurotoxicity with time was small, suggesting that fibrils make little contribution to neurotoxicity. To study the neurotoxicity of diffusible moieties by regulating microenvironments, we created a bio-mimetic microfluidic system generating spatial gradients of diffusible oligomeric assemblies and assessed their effects on cultured neurons. We found amyloid beta exposure produced an atrophy effect and observed neurite extension during the differentiation of neural progenitor cells increased when cells were cultured with continuous flow. The results demonstrate the potential neurotoxicity of oligomeric assemblies and establish a prospective microfluidic platform for studying the neurotoxicity of amyloid beta.
\end{abstract}

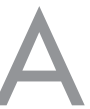

lzheimer's disease, the most prevalent form of dementia in the elderly, is characterized by hallmark lesions - extracellular amyloid plaques ${ }^{1}$. The generation of pathogenic amyloid beta proteins is a widely known example of regulated intramembrane proteolysis, a physiological mechanism ${ }^{2}$. In this process, pathogenic amyloid beta proteins are sequentially cleaved from the amyloid precursor protein by two enzymes, $\beta$-secretase and $\gamma$-secretase (Fig. 1a). The proteins are liberated into the brain interstitial fluid ${ }^{3}$, including the synaptic cleft, after proteolytic processing in the endoplasmic reticulum. Because amyloid beta proteins have a propensity to polymerize, aggregation subsequently occurs, leading to the formation of oligomeric species of amyloid beta proteins that are small enough to diffuse through the interstitial fluids. Amyloid beta monomers tend to aggregate and polymerize, forming oligomers, which in turn, contribute to protofibrils, and the protofibrils to fibrils (Fig. 1b). Although there is a general consensus that amyloid beta induces Alzheimer's disease, there has been a controversy surrounding the question of which molecular entity of amyloid beta is most toxic and plays a principal role in causing the disease. Early studies argued that the pathogenicity of amyloid beta derived from the aggregation process $^{4}$, suggesting that fibrils-the end-products of protein aggregation-are the direct cause of the neurotoxicity that drives Alzheimer's disease ${ }^{5,6}$. However, evidence for induction of cellular degeneration by nonfibrillar amyloid beta protein ${ }^{7}$ indicates the potential neurotoxicity of oligomeric amyloid beta. Moreover, it was found that inert fibrillar aggregates protect against amyloid beta toxicity rather than inducing neurotoxicity $^{8,9}$. In fact, numerous recent studies ${ }^{10-13}$ have demonstrated that soluble protein oligomers possess neurotoxicity; thus, soluble oligomeric assemblies of amyloid beta are now believed to be the moieties crucial for Alzheimer's disease initiation. Several studies have suggested that immunization with amyloid beta peptide reduces neuropathologic features of Alzheimer's disease ${ }^{14}$, highlighting the importance of developing a vaccine targeting an immunogenic self-epitope of the most toxic amyloid beta moiety ${ }^{13}$.

As discoveries that monomers are innocuous have been widely known ${ }^{15}$, we excluded this moiety. Our study focused on determining which form of amyloid beta (oligomeric assemblies or fibril) is the most toxic to neuron cells. We noted that amyloid beta aggregation is time-dependent, and thus, the resulting neurotoxicity is regarded as the accumulative effect with time. We first analyzed the size distributions of a number of fibrils over time using an Electrical Sensing Zone system based on the Coulter principle, in which production of a voltage across an aperture displaces its own volume of conducting liquid. We analyzed the neurotoxicity of different sets of amyloid 

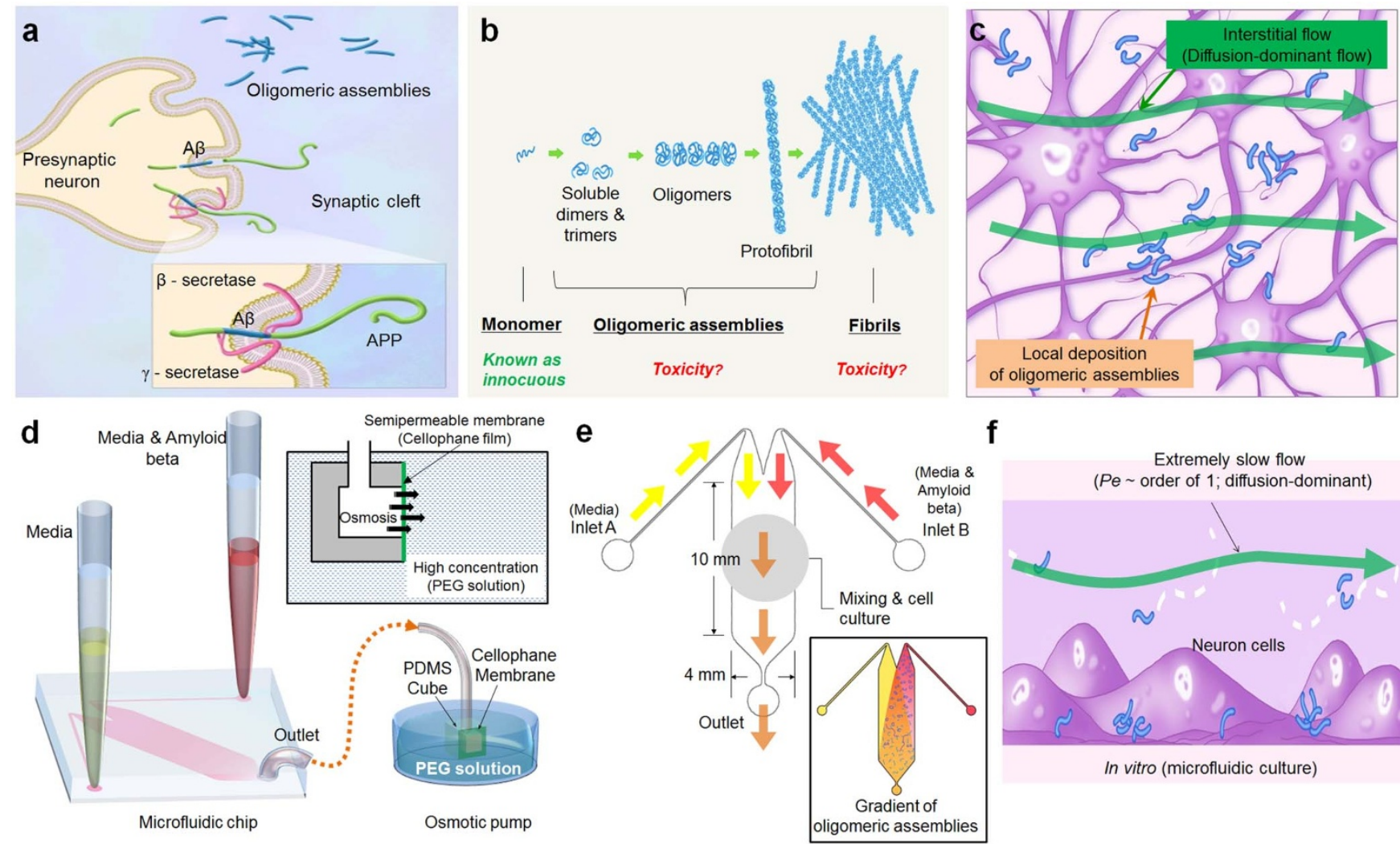

f

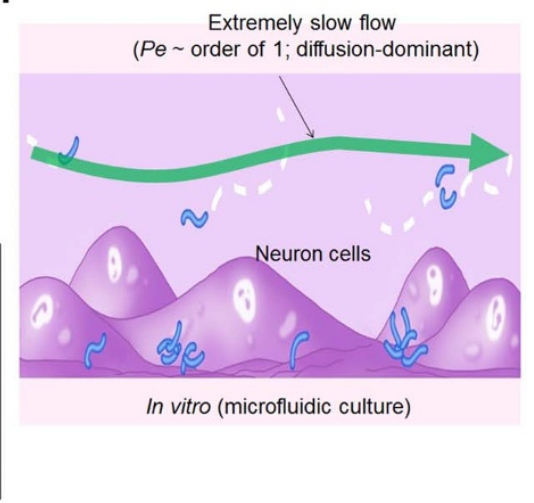

Figure 1 Concept and design of microfluidic system. (a) Oligomeric assembly diffusion in the synapse. (b) Oligomeric assemblies and fibrils are both suspected neurotoxic entities. (c) Neuronal cells exposed to a gradient of oligomeric assemblies. (d) Experimental setup of microfluidic system. Inset: osmotic flow is created by the pump unit. (e) Schematic diagram of the microfluidic system. Small inset: oligomeric assembly gradient.

(f) Interstitial level of slow flow.

beta whose incubation time was different, to find the correlation between an increased number of fibrils and neurotoxicity.

The blood-brain barrier (BBB) restricts the amyloid beta transport from brain to blood or vice versa due to tight junctions of the cerebrovascular endothelial cells ${ }^{16}$. Exchange of amyloid beta through $\mathrm{BBB}$ is a receptor-mediated process which is dependent on RAGE (receptor for advanced glycation endproducts), a transmembrane receptor of immunoglobulin super family (Supplementary Fig. 1). Since RAGE expression in the vascular system is highly dependent on the concentration of amyloid beta ${ }^{17}$ and differing in each species, intraparenchymal injection is used in vivo model ${ }^{18}$, so that the effect of RAGE-mediated amyloid beta transport is excluded. For real-time observation of cellular responses to amyloid beta, direct exposure of amyloid beta to the neuron cells was conducted conventional in vitro model ${ }^{19,20}$, however such conventional culture systems, which are unable to recapitulate oligomeric amyloid beta gradients and continuous flow conditions, have a limited ability to mimic the in vivo fluidic environment of brain tissue. Although the general physiological mechanism of amyloid beta has drawn considerable research interest, a reliable method for studying it under a well-regulated microenvironment has been challenging. To overcome this limitation, we introduce a microfluidic approach for quantitatively analyzing the effect of oligomeric amyloid beta on neurons within an in vivo-like microenvironment (Fig. 1c). The resulting bio-microfluidic chip embodies the conditions under which proteolysis occurs, providing osmosis-driven low-speed flow $(0.25 \mu \mathrm{L} / \mathrm{min})$ comparable to interstitial flow rates $(0.1-0.3 \mu \mathrm{L} / \mathrm{min} \cdot \mathrm{g})^{21}$, and allowing the creation of standing gradients of test molecules. Both the Reynolds number (inertia vs. viscous effect) and the Peclet number (convection vs. diffusion effect) are small; therefore, the flow is laminar with diffusion-dominant flow characteristic in which diffusion mixing at the interface of laminar streams occurs and lasts for a prolonged time (days to weeks). The microfluidic chip enables the generation of gradients slow enough to allow diffusion at the interface between two separate fluid phases. An interstitial flow speed of only a few micrometers per second generated in the microfluidic chip avoids shear stress ${ }^{22}$. By generating flow and gradient of oligomeric assemblies of amyloid beta continuously, our platform perfectly simulates an in vivo condition where neuron cells were bathed and processed after cleavage of amyloid beta, which is an example of regulated intramembrane proteolysis (RIP), a convergence physiological mechanism. To our knowledge, our study is the first quantitative analysis for the accumulative effect of time-dependent amyloid beta aggregation and the effect of physiological slow flow on neuronal cell survival under amyloid beta concentration in a biomimetic microenvironment.

\section{Results}

Mimicking the in vivo environment using a microfluidic system. To provide an in vitro strategy for investigating the neurotoxicity of oligomeric assemblies of amyloid beta following diffusion into the brain interstitium, we use a microfluidic culture system that can create extremely slow flow rates comparable to those of interstitial flow in brain tissue. The microfluidic culture system is composed of a patterned polydimethylsiloxane (PDMS) mold bonded to a glass coverslip, a flexible tube, an osmotic pump, and a $0.05 \mathrm{M}$ polyethylene glycol (PEG) solution (Fig. 1d and Supplementary Fig. 2-4, Supplementary Fig. 3 and 4 illustrating soft lithography and fabrication method of osmosis pump). The dimensions of the main channel of the chip are $4 \mathrm{~mm}$ (width) $\times 10 \mathrm{~mm}$ (length) $\times$ $200 \mu \mathrm{m}$ (height). The driving force of the flow is generated by the osmotic pump, located inside a Petri dish filled with PEG solution, which consists of a cubic deionized water-filled PDMS chamber with 
one cellulose membrane window ${ }^{23}$ (right, Fig. 1d); the difference in concentration between water and PEG solution at the cellulose membrane of the osmotic pump creates osmotic pressure, which is the main driving force for the flow of media. Neuronal cells were allowed to adhere in the main channel of the chip and were maintained for 7 days after plating by culturing with a continuous flow of pure media. For the last 3 days, pure media and amyloid beta dissolved in media (final concentration, $5 \mu \mathrm{M}$ ) were introduced via two respective inlet ports, creating a concentration gradient in the main channel. At the interface where the two solution streams meet, diffusion occurs; therefore, a patterned gradient is generated and its profile covers the main channel of the chip (Fig. 1e). Diffusiondominant flow is generated and maintained for a long time in the main channel of the chip (Fig. 1f). Within 7 days after seeding neural progenitor cells in the main chamber with continuous flow, in vitro differentiation and neurite extension of neural progenitor cells was fully completed. For supportive visualization of the generated concentration profile, a three-dimensional fluid dynamics model was implemented with the same microchannel geometry; see the supplementary data for the computational method, corresponding results, and supplementary movie.

Fibrillogenesis of amyloid beta protein (1-42) fragments over time and associated neurotoxicity. We choose a $2.5 \mu \mathrm{M}$ amyloid beta solution for the experiments. At this low concentration solution of amyloid beta, the monomer and oligomeric assembly states are at a free-energy minimum ${ }^{24}$, and therefore, the conversion rate into fibril is slower; see reference for a phase diagram of amyloid beta states ${ }^{24}$. To determine the number of fibrils in a $2.5 \mu \mathrm{M}$ amyloid beta

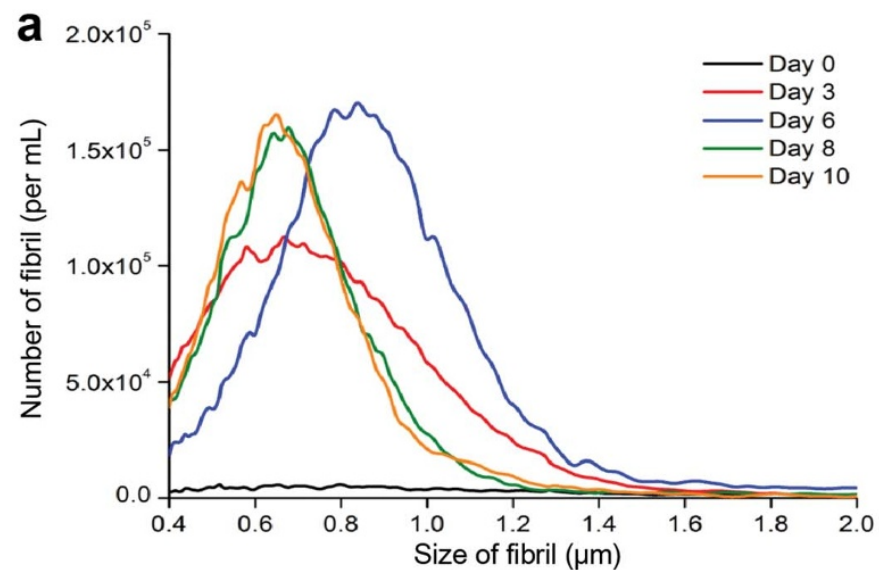

C

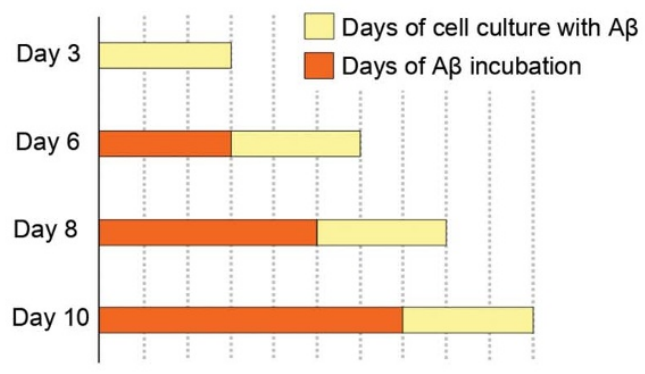

solution, we used an analytical counting equipment (Electrical Sensing Zone system), which exploits an electric field for counting and sizing particles. For this test, we used deionized water as solvent medium since the electrolytes dissolved in culture medium create errors in counting fibrils. We set the detection window from 0.4 to $48 \mu \mathrm{m}$ to count the fibrils because a single protofibril has lengths up to $0.2 \mu \mathrm{m}^{10}$ and at least two protofilbrils are required to form a fibril. Because we wanted to know the change in the size distribution of fibrils, we incubated a $2.5 \mu \mathrm{M}$ amyloid beta solution for different numbers of days. Over time, the amyloid beta moiety underwent dynamic exchange and thereafter was brought into equilibrium for each individual moiety present. During the process of fibril nucleation and growth, fibrils adopted certain length and size distributions (Fig. 2a). Amyloid beta size in solution without incubation was evenly distributed, but was shifted toward the left after incubation. Modal fibril lengths after 3, 6, 8, and 10 days were $0.627,0.769,0.702$, and $0.640 \mu \mathrm{m}$ (peaks of each graph in Fig. 2a), respectively, thus exhibiting little change over time. We postulated that, at a certain concentration of amyloid beta, fibril growth reached a saturation level. The number of fibrils, in volume units, increased over time (Fig. 2b). During the first 6 days, the increase in the number of fibrils was dramatic, whereas the degree of increase was relatively smaller during the last 4 days (day 6 to day 10); overall, however, the number of mature fibrils increased with time indicating amyloid beta proteins have a propensity of self-aggregation and therefore they form fibrils, an end-product of amyloid beta. We exposed primary rat neuronal cells under fixed conditions of amyloid beta in Neurobasal media alone and measured the resulting neurotoxicity by performing an MTT assay. Prenatal rat neuronal cells isolated
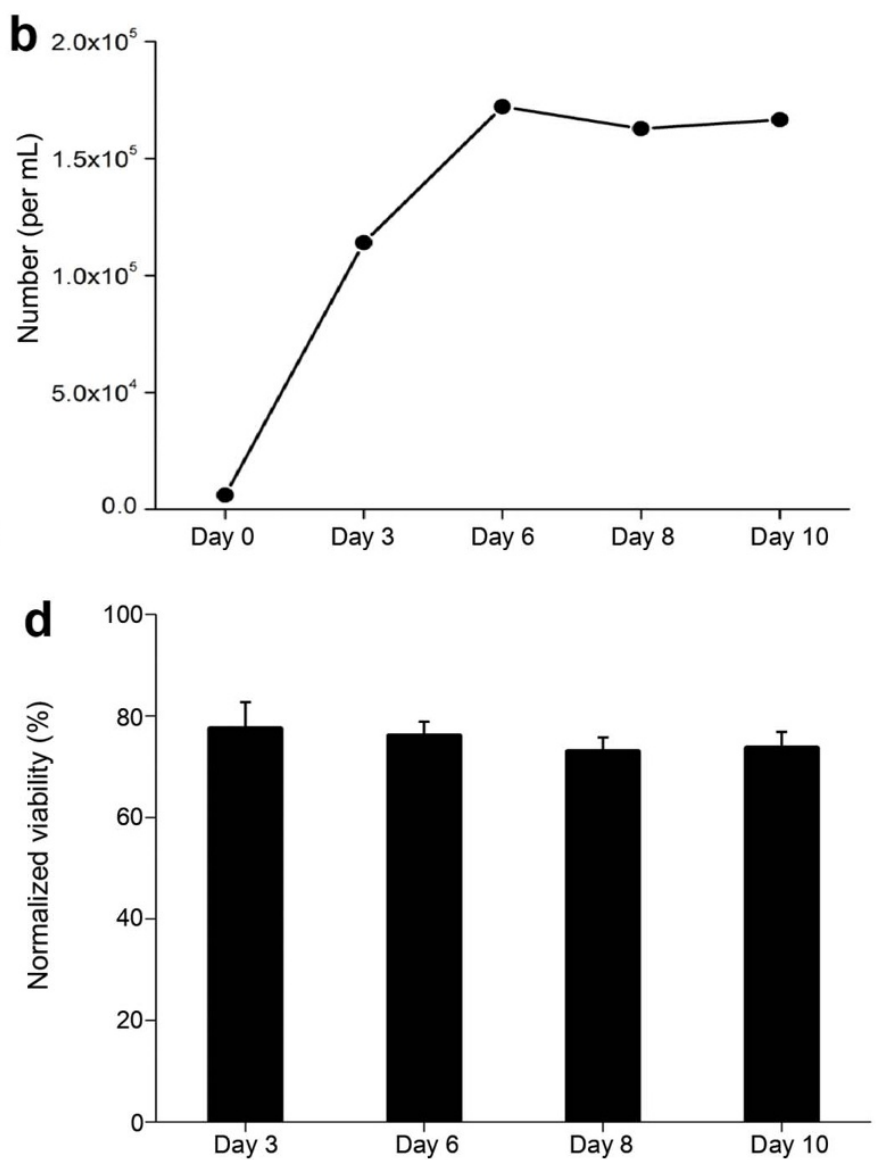

Figure $2 \mid$ Fibrillogenesis of amyloid beta over time. (a) The size distribution of fibrils in a $2.5 \mu \mathrm{M}$ amyloid beta with respect to incubation time. (b) The frequency of fibrils with respect to incubation time. (c) Total days of amyloid beta incubation are the summation of days of cell culture with amyloid beta (yellow) and days of solely incubating amyloid beta (orange). (d) Comparison of neuronal cell viability. One-way ANOVA with Scheffe's post hoc analysis $(p>0.1, \mathrm{~F}=2.087)(\mathrm{n}=6)$. 
from E-16 rat embryos were cultured in Neurobasal media for 7 days (Supplementary Fig. 5 shows that neuronal cells isolated from prenatal rat cerebral cortical regions express cortical layer-specific markers CTIP2 and Tbr1). For this experiment, we prepared 4 different $2.5 \mu \mathrm{M}$ amyloid beta solutions; the difference of the solution is the amyloid beta incubation date $(0,3,5$, and 7 days). Then, neural cells were cultured in each of the prepared amyloid beta solutions for 3 days to assess cell damage; graphs in Fig. 2c are made based on the days of cell culture with amyloid beta (yellow) \& the days of solely incubating amyloid beta (orange). It is important to note that amyloid beta aggregation continues even during the 3 days of exposure (Fig. 2c). The results, as shown in Fig. 2d, indicate that the neurotoxicity of amyloid beta changed little with different times. We postulated that cells are damaged by oligomeric assemblies because the change of fibrils in first 6 days was not reflected in the cell viability (see Day 3 to Day 6 in Fig. 2d) and also the cell viability in fibril-rich conditions (Day 6 to Day 10 in Fig. 2d) did not change statistically; in other words, the appearance of fibrils over time did not have a crucial impact on neurotoxicity, implying the damage was done by oligomeric assemblies. It should be noted that in general the conversion rate into oligomeric assemblies is accomplished faster than that into fibril, and therefore, the 3 days are long enough to have a saturated oligomeric assemblies ${ }^{25}$.

On-chip neurotoxicity of amyloid beta oligomeric assemblies. To verify the gradient profiles of oligomeric assemblies in our microchannel, neuronal cells, cultured in our system for 10 days were treated with the Live/Dead assay reagent and immunostained for synapsin II and caspase-3. For the first 7 days, cells were cultured with continuous media flow but without amyloid beta; after 3 days, an oligomeric assembly gradient was added. A pure media was loaded into port A and $5 \mu \mathrm{M}$ solution of amyloid beta was loaded into inlet port B (Fig. 3a). At the interface of the two laminar streams, the amyloid beta solution and the pure media diffused and mixed together, generating concentration gradient profiles that were maintained for 3 days for which the neuronal cells were affected by amyloid beta concentration. Fluorescence images of neuronal cells were taken to live cells (green) and dead cells (red) (left, Fig. 3b), synapsin II (red) and DAPI (blue) (left, Fig. 3c), and caspase-3 (green) and DAPI (blue) (left, Fig 3d). The main channel was divided into 10 sections along the flow; sections exhibiting a stable gradient were defined from 1-5 (Fig. 3a). The green fluorescence from live cells in each section was plotted (right, Fig. 3b) and the slope of the linear fit of the plot from sections $1-5$ were $3.19,3.07$, $2.77,2.70$, and 2.5 , respectively, with the intensity showing a decreasing trend. It shows that the live cell distribution is in accordance with the concentration of amyloid beta, as amyloid beta concentration gets even as flow goes downstream. Further, the absolute value (y-intercept) of the slopes diminished from sections 1-5. This verifies the neurotoxicity of amyloid beta, as cells directly exposed to amyloid beta demonstrated a lower green fluorescent intensity. The red fluorescence from synapsin II also shows a decreasing trend which indicates neuritic disruption is induced by amyloid beta (right, Fig. 3c). The green fluorescence from caspase-3 shows an increasing trend (right, Fig. 3d) since caspase-3 responsible for the apoptosis was highly expressed in the neuronal cells directly exposed to amyloid beta. It indicates the induction of apoptosis by amyloid beta.

We next set up three experimental groups to analyze the impact of flow and amyloid beta on neuron cells. Group 1 (flow/no amyloid beta) consisted of primary neural progenitor cells in the microfluidic chip under continuous fluid flow for 10 days without amyloid beta.

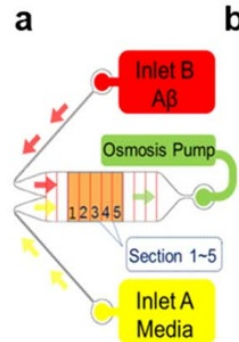

e

\begin{tabular}{|c|c|c|c|}
\hline Experiment \# & \multicolumn{2}{c|}{ Group 1 } & \multicolumn{2}{c|}{ Group 2 } & Group 3 \\
\hline Flow & Yes & Yes & No \\
\hline Amyloid beta & No & Yes & No \\
\hline $\begin{array}{c}\text { Typical } \\
\text { morphology }\end{array}$ & & & \\
\hline & & & \\
\hline Optical image & & & \\
\hline
\end{tabular}

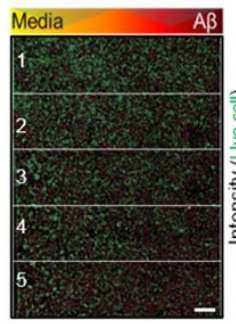

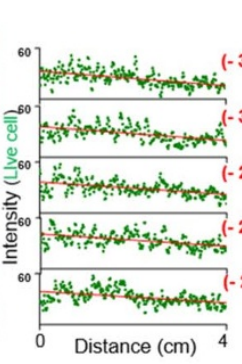

C

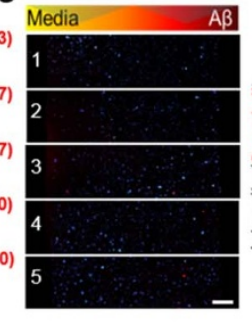

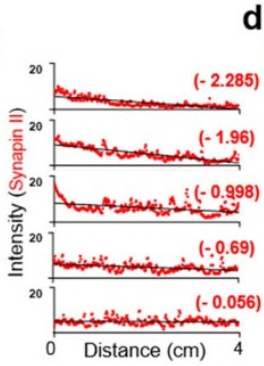

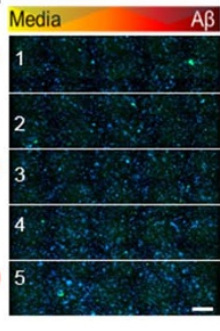

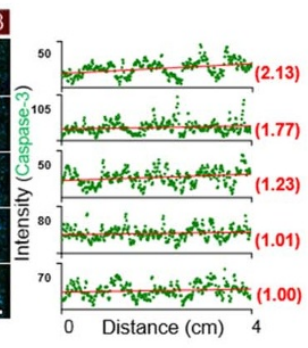

f

g

h
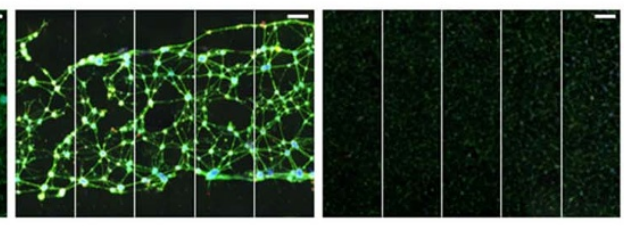

$\mathbf{k}$

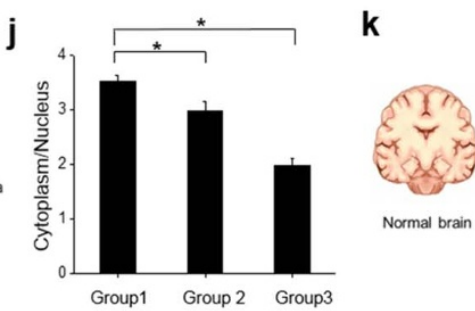

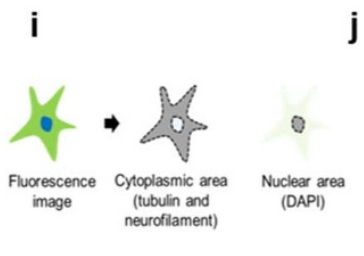

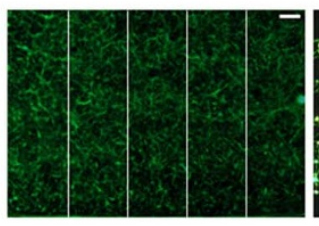

i . 
Group 2 (flow/amyloid beta) was similar to group 1 for the first 7 days, but for the last 3 days, $5 \mu \mathrm{M}$ amyloid beta was added to generate gradient profiles of oligomeric assemblies in the main channel of the chip. Group 3 (no flow/no amyloid beta) was identical to group 1 with the exception of fluid flow. Fig. 3e exemplifies the results obtained after different treatments in the three sets of experiments. Fluorescence micrography (Fig. 3f-h) after immunostaining for tubulin, neurofilament, and nuclei showed that primary neural progenitor cells under continuous media flow for 10 days (Group 1, Fig. 3f) formed robust neural networks and exhibited numerous axons and dendrites around the soma, whereas neuron cells exposed to the gradient of amyloid beta oligomeric assemblies (Group 2, Fig. 3g) showed neuronal loss and destruction of matured axons and dendrites. Primary neural progenitor cells cultured without flow (Group 3, Fig. 3h) showed weak extension of neurites and tended to aggregate. To quantitatively evaluate the three sets of experiments, we used sections 1-5 from each set, as depicted in the images in Fig. 3e (a: group 1, b: group 2, c: group 3), and applied a computerized image analysis to quantify the area ratio of cytoplasm (sum of tubulin and neurofilament) to nucleus. Fig. 3i explains how this was accomplished by integrating the intensity of the cytoplasmic marker tubulin and neurofilament (green fluorescence and red fluorescence) and nuclear marker DAPI (blue fluorescence) of the cells through the designated section of the main channel. As shown in Fig. 3j, the decrease in the ratio of cytoplasm to nucleus of group 2 compared to that of group 1 indicates atrophy, a pathological feature of Alzheimer's disease ${ }^{26}$ (Fig. 3k), validating the possibility of conducting related research using our system. Neural progenitor cells cultured without media flow had the lowest ratio of cytoplasm to nucleus shown in Fig. 3j, indicating that continuous media flow enhanced differentiation and neurite extension of neural progenitor cells. We speculate that continuous media flow in our system facilitates the delivery of oxygen and nutrients, thereby promoting differentiation and neurite extension.

We also performed Live/Dead assays to determine cell viability in the 3 experimental groups. As shown in Fig. 4a, notably, the percentage of viable cells cultured with oligomeric assemblies decreased approximately $20.5 \%$ compared to cells not treated with amyloid beta. The viability of cells cultured with media flow was $7.5 \%$ greater than that of cells cultured without media flow. To validate viability tests in the chip after exposure to amyloid beta, we performed a flow cytometry analysis on neurons cultured in 6-well plates. We used 6 different concentrations of amyloid beta $(1.5,2.0,2.5,3.0,3.5$, and $4.0 \mu \mathrm{M})$. Apoptosis induced by amyloid beta results in structural changes such as compaction of nuclear chromatin, blebbing of membrane, and DNA fragmentation (Fig 4d). We measured cellular DNA content in subG1, G0/G1, S and G2/M to determine the distribution of propidium iodide (PI)-stained cells in each phase (Fig. 4b). We observed an increased accumulation of the sub-G1 (apoptotic) cell population after treating with amyloid beta for 3 days. The values for decreased viability of neuronal cells after amyloid beta treatment compared to cells not treated with amyloid beta are presented in Fig. 4c. Although there were minor differences in the percentages of viable cells between cells cultured in-chip and in 6-well plates, considering that cell viability increases with media flow, the in-chip results are reliable. Above-mentioned harmful effect of amyloid beta appeared also in $3 \mathrm{D}$ neurospheres exposed to amyloid beta ${ }^{27}$ (see Supplementary Fig. 6).

\section{Discussion}

Recent studies have demonstrated that before the complete accumulation of amyloid beta, intermediate moieties such as oligomeric assemblies become toxic to neuronal cells. Deleterious effects of oligomeric assemblies on synaptic functions result in progressive neuronal changes and oxidative injury ${ }^{28}$. However, assessments of the neurotoxicity of oligomeric assemblies are unreliable because of the difficulty in selectively separating oligomeric assemblies from the amyloid beta solution. In this study, we found little correlation between increases in the number of fibrils and neurotoxicity (Fig. 2d). Due to the nature of self-assembly of amyloid beta, the monomer and oligomeric assemblies are consumed in the production of a given moiety. Therefore, the decrease in the number of monomers or oligomeric assemblies reflects the increase in other moieties. However, it is possible that, at a certain concentration, the numbers of oligomeric assemblies is maintained rather than a

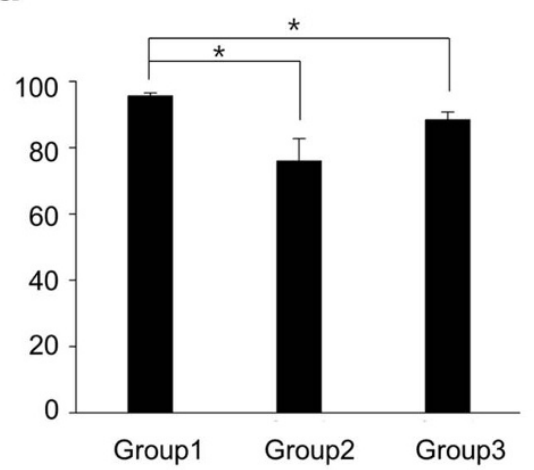

b
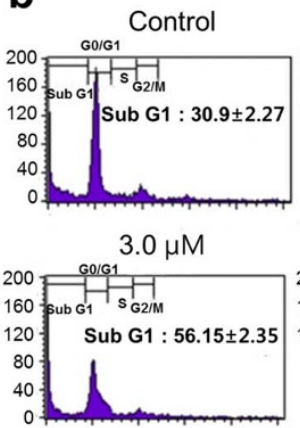

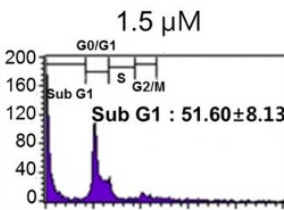

$3.5 \mu \mathrm{M}$
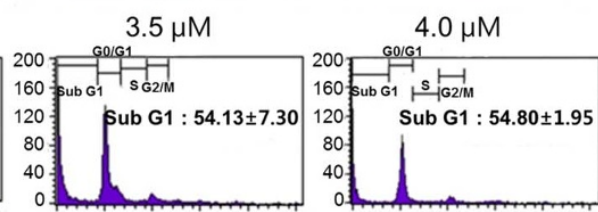
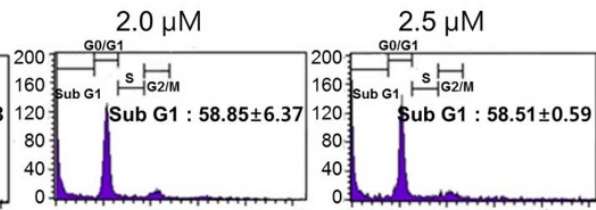

(1)
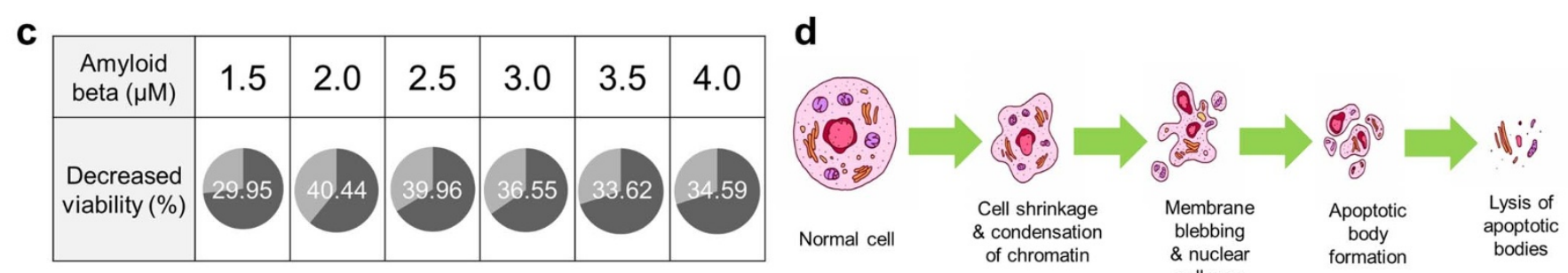

Figure $4 \mid$ Neurotoxicity of oligomeric assemblies assessed both in microfluidic chip and conventional platform. (a) A comparison of viability within a chip. $\left({ }^{*} p<0.001\right.$, two-tailed test) $(\mathrm{n}=20)$. (b) FACS analysis of the neurotoxicity of amyloid $(\mathrm{n}=3)$. (c) Decreased viabilities of neuronal cells after exposure of amyloid beta compared to cells without amyloid beta. (d) Apoptosis induced by amyloid beta. 
increased because oligomeric assemblies are also consumed to make fibrils. It was reported that there is an equilibrium state (a triple point) of monomer, oligomeric assemblies, and fibril depending on their relative concentration ${ }^{24}$. In other words, the number of oligomeric assemblies is at equilibrium and, therefore, might be relatively unchanged. This postulated maintenance in the number of oligomeric assemblies is based on our experimental results, which showed little change in cell viability over a time frame that saw increases in the number of fibrils. It is oligomeric assemblies-potential toxic moieties-that maintain their number over time, a phenomenon that results in negligible changes in cell viability.

Interstitial fluid in the brain has a major impact on the pathology and physiology of neural cell ${ }^{21}$. Because the role of interstitial fluid in controlling amyloid beta metabolism has not been clarified, the dynamics of interstitial fluid flow in the brain should be closely investigated. Our platform is capable of facilitating such studiesnot only mimicking the physiological conditions of the brain, but also helping to understand the mechanism of amyloid beta under interstitial fluid flow conditions ${ }^{29}$. Embodiment of a slow flow rate corresponding to the rate of interstitial flow simulates how cleaved pathogenic amyloid beta proteins diffuse through the interstitial fluid and have a deleterious impact on neuronal cells (Fig. 3 and Fig. 4).

A number of studies have emphasized the importance of amyloid beta immunization because of its potential to mitigate the pathological features of Alzheimer's disease. In a transgenic mouse model of Alzheimer's disease, immunization with amyloid beta was shown to eliminate the accumulation of amyloid deposits ${ }^{30}$ and associated cognitive impairment ${ }^{31}$. Furthermore, compared with unimmunized Alzheimer's disease patients, immunized patients show a reduction in diagnostic neuropathological features of Alzheimer's disease ${ }^{32}$, indicating the importance of immunization with amyloid beta. However, the design of amyloid beta vaccines is problematic in that the question of which moieties of amyloid beta are most toxic, and thus the appropriate target of a vaccine for Alzheimer's disease, remains unresolved ${ }^{33}$. Our results demonstrate a weak correlation between the increase in the number of fibrils with time and neurotoxicity, indicating the potential neurotoxicity of oligomeric assemblies and suggesting that the emphasis for vaccine epitope modeling should be placed on oligomers rather than fibrils. On the basis of the results presented here, we suggest that a conformation-specific oligomer-binding antibody should be developed.

In summary, the increase in the number of fibrils does not play a significant role in showing accumulative neurotoxic effects on the cells indicating potential neurotoxicity of oligomeric assemblies rather than fibrils. We believe the fibril accumulation is just an end product and not directly linked to the neuronal cell's viability, although it is a definite indicator for a developmental stage of Alzheimer's disease. Thus future treatment techniques should be focused in terms of prevention of the oligomeric assemblies. Our microfluidic culture platform provides a useful tool for analyzing the neurotoxicity of oligomeric assemblies of amyloid beta in a bio-mimetic setting and will greatly facilitate other studies on regulated intramembrane proteolysis. Our platform will bridge the gaps between the traditional in vitro cell culture methods with the in vivo intraparenchymal injections model. In contemplating future perspectives, the application of the proposed long-term slow-flow microfluidic system to neurons and neural progenitors will enable long-term in vitro observation on the neural differentiation, development of brain, onset of Alzheimer's disease, and neural degeneration. In addition, biochemical combinations and their diffusion-dominant delivery process to neural cells should be the target issues in development of new treatments for Alzheimer's disease, for which our microfluidic system should be an ideal platform to facilitate such studies.

\section{Methods}

Microfluidic gradient chip fabrication. The microfluidic gradient chip made of PDMS was fabricated by photolithography and replica molding using photoresist patterns as master templates. SU-8, a negative photoresist on a silicon wafer, was patterned by photolithography, and $200-\mu \mathrm{m}$ deep main channels were generated. After the photolithography process, the photoresist pattern was developed and washed to clear debris. Well-mixed PDMS prepolymer and curing agent (Sylgard 184; Dow Corning, Midland, MI, USA) were poured onto the master template and cured in an oven at $120^{\circ} \mathrm{C}$ for $1 \mathrm{~h}$. The PDMS plate was then peeled from the master template, and two inlets and an outlet were punched with a sharp needle. The glass coverslips were cleaned using acetone, isopropyl alcohol and 70\% alcohol, and then the surfaces of the PDMS plate and glass coverslip were oxidized by $\mathrm{O}_{2}$ plasma using a plasma reactor. After binding the PDMS piece to the glass coverslip, the device was kept at $90^{\circ} \mathrm{C}$ for $1 \mathrm{~h}$ to allow complete annealing.

Analysis of amyloid beta particle size. The size distribution of fibrils over time was analyzed by first incubating a solution of synthetic amyloid beta protein (1-42) (Sigma-Aldrich, St. Louis, MO, USA) at $37^{\circ} \mathrm{C}$ in an atmosphere of $5 \% \mathrm{CO}_{2}$ for $3,6,8$, or 10 days. A Multisizer 4 Coulter Counter (Beckman Coulter, Brea, CA, USA) employing the Coulter Principle, also known as the Electrical Sensing Zone (ESZ) method, was used for particle counting.

Cell proliferation assay. Prenatal rat neuronal cells isolated from E-16 rat embryos were seeded at a density of $5 \times 10^{4}$ cells/well into 96-well plates and cultured in Neurobasal media for 7 days. Cells were subsequently incubated with $2.5 \mu \mathrm{M}$ synthetic amyloid beta protein (1-42) (Sigma-Aldrich). MTT (3-(4,5dimethylthiazol-2-yl)-2,5-diphenyltetrazolium bromide) solution $(10 \mu \mathrm{L})$ was added to each well, and the cells were further incubated at $37^{\circ} \mathrm{C}$ for $3 \mathrm{~h}$. Absorbance at $480 \mathrm{~nm}$ was measured using a Molecular Devices SpectraMax 250 Microplate Reader (GMI, Minneapolis, MN, USA).

Culture of primary neural progenitor cells in a microfluidic gradient chip. Neuronal cells were isolated from prenatal cerebral cortical regions of rat embryos (embryonic day 16) using surgical procedures approved by the Institutional Animal Care and Use Community (IACUC) of Texas Southern University. A $200-\mu \mathrm{L}$ suspension of cells $\left(2 \times 10^{6}\right.$ cells $\left./ \mathrm{mL}\right)$ was loaded into a microchannel coated with PLO (Poly-L-Ornithine) $(50 \mu \mathrm{g} / \mathrm{mL})$ and laminin $(5 \mu \mathrm{g} / \mathrm{mL})$. Attached neural progenitor cells were maintained in Neurobasal media (Gibco) supplemented with B-27 Supplement (Gibco), $0.5 \mathrm{mM}$ L-glutamine, and 100 units/mL penicillin for 7 days, and then exposed to $2.5 \mu \mathrm{M}$ amyloid beta for an additional 3 days.

Cell viability test. The viability of neuronal cells cultured in a microfluidic gradient chip was assessed using a Live/Dead assay kit (Invitrogen, Calsbad, CA, USA) as described by the manufacturer. Briefly, $1 \mu \mathrm{L}$ of calcein AM solution and $5 \mu \mathrm{L}$ of ethidium homodimer- 1 solution dissolved in $2 \mathrm{~mL}$ of neurobasal media were added to a microfluidic gradient chip via the outlet port. The cells were incubated at $37^{\circ} \mathrm{C}$ for $40 \mathrm{~min}$, and stained cells were examined using a fluorescence microscope (AxioVision 4; Zeiss, Oberkochen, Germany).

Immunocytochemistry. Rat neural progenitor cells seeded in a microfluidic gradient chip and 6-well plates were fixed in $4 \%$ paraformaldehyde for $20 \mathrm{~min}$ at $4{ }^{\circ} \mathrm{C}$ and rinsed with $0.1 \%$ bovine serum albumen (BSA) in phosphate-buffered saline (PBS). The cells were then incubated in $0.1 \%$ Triton X-100 in PBS for $20 \mathrm{~min}$ at room temperature and, after rinsing with $0.1 \%$ BSA in PBS, were blocked for $30 \mathrm{~min}$ at $4{ }^{\circ} \mathrm{C}$. For primary antibodies, we used antibodies to Neurofilament $\mathrm{H}$ (Millipore), Beta III tubulin (Invitrogen), Ctip2 (Abcam), TBR1 (Abcam), Synapsin II (Abcam), Caspase3 (Abcam) and Tuj1 (Covance MMS-435P, PRB-435P). Cells were rinsed with $0.1 \%$ BSA in PBS, and then probed with Alexa Fluor 488-conjugated anti-mouse and Alexa Fluor 594-conjugated anti-rabbit IgG (both from Invitrogen) for $90 \mathrm{~min}$ at $4{ }^{\circ} \mathrm{C}$. Fluorescent images of neuron cells cultured in microfluidic chip were collected using a fluorescence microscope (AxioVision 4; Zeiss) while those of neuron cells cultured in 6-well plates were obtained using a confocal microscope (LSM 5 Exciter; Zeiss) after counterstaining nuclei with 4,6-diamidino-2-phenylindole dihydrochloride (DAPI; Invitrogen).

Flow cytometric analysis of apoptosis. Neuronal cells $\left(2.2 \times 10^{6}\right.$ cells $\left./ \mathrm{mL}\right)$, cultured for 7 days in 6-well plates, were exposed to $2.5 \mu \mathrm{M}$ amyloid beta for 3 days and then harvested by trypsinization. Thereafter, neuronal cells were washed with PBS and fixed/stored with $90 \%$ ethanol at $-20^{\circ} \mathrm{C}$ until the day of analysis. The cells were incubated with an RNase A solution in PBS (30 $\mu \mathrm{g} / \mathrm{mL}$; Sigma-Aldrich), and cellular DNA was stained with PI (50 $\mu \mathrm{g} / \mathrm{mL}$; Sigma-Aldrich). DNA content was assessed using a FACSCalibur flow cytometer and analyzed with FACSCalibur and CellQuest software (BD Biosciences, San Diego, CA, USA).

Statistical analysis. All quantitative data were expressed as means \pm standard errors of the mean (SEM). All collected data were analyzed using unpaired t-tests or one-way analyses of variance (ANOVA) with Scheffe's post hoc test using the statistical software SPSS, Version 10.0 (SPSS, Chicago, IL, USA). P-values below 0.001 were considered statistically significant. 
1. Probst, A., Langui, D. \& Ulrich, J. Alzheimer's disease: a description of the structural lesions. Brain Pathology 1, 229-239 (1991).

2. Brown, M. S., Ye, J., Rawson, R. B. \& Goldstein, J. L. Regulated Intramembrane Proteolysis: Review A Control Mechanism Conserved from Bacteria to Humans. Cell 100, 391-398 (2000).

3. Cirrito, J. R. et al. In vivo assessment of brain interstitial fluid with microdialysis reveals plaque-associated changes in amyloid- $\beta$ metabolism and half-life. The Journal of neuroscience 23, 8844-8853 (2003).

4. Bucciantini, M. et al. Inherent toxicity of aggregates implies a common mechanism for protein misfolding diseases. Nature 416, 507-511 (2002).

5. Lorenzo, A. \& Yankner, B. Amyloid Fibril Toxicity in Alzheimer's Disease and Diabetesa. Annals of the New York Academy of Sciences 777, 89-95 (1996)

6. Lorenzo, A. \& Yankner, B. A. Beta-amyloid neurotoxicity requires fibril formation and is inhibited by congo red. Proceedings of the National Academy of Sciences 91 , 12243 (1994).

7. Zhu, Y. J., LIN, H. \& LAL, R. Fresh and nonfibrillar amyloid ß protein (1-40) induces rapid cellular degeneration in aged human fibroblasts: evidence for AßPchannel-mediated cellular toxicity. The FASEB Journal 14, 1244-1254 (2000).

8. Pallitto, M. M., Ghanta, J., Heinzelman, P., Kiessling, L. L. \& Murphy, R. M. Recognition sequence design for peptidyl modulators of $\beta$-amyloid aggregation and toxicity. Biochemistry 38, 3570-3578 (1999).

9. Zetterberg, H., Blennow, K. \& Hanse, E. Amyloid $\beta$ and APP as biomarkers for Alzheimer's disease. Experimental gerontology 45, 23-29 (2010).

10. Hartley, D. M. et al. Protofibrillar intermediates of amyloid $\beta$-protein induce acute electrophysiological changes and progressive neurotoxicity in cortical neurons. The Journal of neuroscience 19, 8876-8884 (1999).

11. Lashuel, H. A., Hartley, D., Petre, B. M., Walz, T. \& Lansbury, P. T. Neurodegenerative disease: amyloid pores from pathogenic mutations. Nature 418, 291-291 (2002).

12. Lambert, M. et al. Diffusible, nonfibrillar ligands derived from A $\beta 1-42$ are potent central nervous system neurotoxins. Proceedings of the National Academy of Sciences 95, 6448 (1998).

13. Klein, W. L., Krafft, G. A. \& Finch, C. E. Targeting small A [beta] oligomers: the solution to an Alzheimer's disease conundrum? Trends in neurosciences 24, 219-224 (2001).

14. Janus, C. et al. Ab peptide immunization reduces behavioural impairment and plaques in a model of Alzheimer's disease. Nature 408, 979-981 (2000).

15. Klein, W. L. A [beta] toxicity in Alzheimer's disease: globular oligomers (ADDLs) as new vaccine and drug targets. Neurochemistry international 41, 345-352 (2002).

16. Begley, D. J. \& Brightman, M. W. Structural and functional aspects of the bloodbrain barrier. Progress in drug research 61, 39-78 (2003).

17. Du Yan, S. et al. RAGE and amyloid- $\beta$ peptide neurotoxicity in Alzheimer's disease. Nature 382, 685-691 (1996).

18. Stepanichev, M. Y., Moiseeva, Y. V., Lazareva, N., Onufriev, M. \& Gulyaeva, N. Single intracerebroventricular administration of amyloid-beta (25-35) peptide induces impairment in short-term rather than long-term memory in rats. Brain research bulletin 61, 197-205 (2003).

19. Loo, D. T. et al. Apoptosis is induced by beta-amyloid in cultured central nervous system neurons. Proceedings of the National Academy of Sciences 90, 7951-7955 (1993)

20. Mark, R. J., Pang, Z., Geddes, J. W., Uchida, K. \& Mattson, M. P. Amyloid $\beta$-peptide impairs glucose transport in hippocampal and cortical neurons: involvement of membrane lipid peroxidation. The Journal of neuroscience 17 , 1046-1054 (1997)

21. Abbott, N. J. Evidence for bulk flow of brain interstitial fluid: significance for physiology and pathology. Neurochemistry international 45, 545-552 (2004).

22. Park, J. Y. et al. Differentiation of Neural Progenitor Cells in a Microfluidic ChipGenerated Cytokine Gradient. Stem Cells 27, 2646-2654 (2009).
23. Park, J. Y., Hwang, C. M. \& Lee, S. H. Gradient generation by an osmotic pump and the behavior of human mesenchymal stem cells under the fetal bovine serum concentration gradient. Lab Chip 7, 1673-1680 (2007).

24. Schmit, J. D., Ghosh, K. \& Dill, K. What Drives Amyloid Molecules To Assemble into Oligomers and Fibrils? Biophysical Journal 100, 450-458 (2011).

25. Lee, J., Culyba, E. K., Powers, E. T. \& Kelly, J. W. Amyloid- $\beta$ forms fibrils by nucleated conformational conversion of oligomers. Nature Chemical Biology 7, 602-609 (2011)

26. Kesslak, J. P., Nalcioglu, O. \& Cotman, C. W. Quantification of magnetic resonance scans for hippocampal and parahippocampal atrophy in Alzheimer's disease. Neurology 41, 51-51 (1991).

27. Choi, Y. J., Park, J. \& Lee, S.-H. Size-controllable networked neurospheres as a 3D neuronal tissue model for Alzheimer's disease studies. Biomaterials (2013).

28. Ondrejcak, T. et al. Alzheimer's disease amyloid $\beta$-protein and synaptic function. Neuromolecular medicine 12, 13-26 (2010).

29. Park, J. Y., Takayama, S. \& Lee, S.-H. Regulating microenvironmental stimuli for stem cells and cancer cells using microsystems. Integrative Biology 2, 229-240 (2010).

30. Schenk, D. et al. Immunization with amyloid-bold beta attenuates Alzheimerdisease-like pathology in the PDAPP mouse. Nature 400, 173-177 (1999).

31. Morgan, D. et al. A $\beta$ peptide vaccination prevents memory loss in an animal model of Alzheimer's disease. Nature 408, 982-985 (2000).

32. Nicoll, J. A. R. et al. Neuropathology of human Alzheimer disease after immunization with amyloid- $\beta$ peptide: a case report. Nature medicine $9,448-452$ (2003).

33. Klein, W. L., Krafft, G. A. \& Finch, C. E. Targeting small A $\beta$ oligomers: the solution to an Alzheimer's disease conundrum? Trends in neurosciences $\mathbf{2 4}$ 219-224 (2001)

\section{Acknowledgements}

This study was supported by the Conversing Research Center Program through the National Research Foundation of Korea (NRF) funded by the Ministry of Education, Science and Technology (2012K001360 and 2012R1A1A1015181) and the National Research Foundation of Korea (NRF) grant funded by the Korea government (MEST) (No. 2012026340) and the Industrial Core Technology Development Program funded (No. 10041923) by the Ministry of Knowledge Economy.

\section{Author contributions}

Y.J. Choi designed and performed research and wrote the paper; S.Chae made analytical study; J.H. Kim assisted experiments; K.F. Barald consulted on the manuscript; J.Y. Park and S.H. Lee proposed the idea, managed the research process and wrote the paper.

\section{Additional information}

Supplementary information accompanies this paper at http://www.nature.com/ scientificreports

Competing financial interests: The authors declare no competing financial interests.

License: This work is licensed under a Creative Commons Attribution-NonCommercial-NoDerivs 3.0 Unported License. To view a copy of this license, visit http://creativecommons.org/licenses/by-nc-nd/3.0/

How to cite this article: Choi, Y.J. et al. Neurotoxic amyloid beta oligomeric assemblies recreated in microfluidic platform with interstitial level of slow flow. Sci. Rep. 3, 1921; DOI:10.1038/srep01921 (2013) 